\title{
Learning-Induced Changes in mPFC-BLA Connections After Fear Conditioning, Extinction, and Reinstatement of Fear
}

\author{
Rose-Marie Vouimba' and Mouna Maroun*,2 \\ 'Université Bordeaux I, Institut de Neurosciences Cognitives et Intégratives d'Aquitaine (INCIA), CNRS UMR 5287, Avenue des Faculté 33405, \\ Talence, France; ' Department of Neurobiology, Faculty of Natural Sciences, University of Haifa, Haifa, Israel
}

\begin{abstract}
The neural circuit linking the medial prefrontal cortex (mPFC) and the basolateral amygdala (BLA) has crucial roles in both the acquisition and the extinction of fear. However, the mechanism by which this circuit encodes fear and extinction remains unknown. In this study, we monitored changes in the magnitude of evoked field potentials (EFPs) in the mPFC-BLA and BLA-mPFC pathways following auditory fear conditioning and extinction, in freely moving rats. We report that extinction of fear is mediated by depression of the EFPs in the $\mathrm{mPFC}-\mathrm{BLA}$ and by potentiation in the reciprocal pathway of BLA-mPFC. Interestingly, reinstatement of fear was associated with recovery of freezing and with reversal of the changes in EFPs that were observed following extinction in both pathways. The findings indicate that the mPFC-BLA circuit expresses differential changes following fear and extinction and point to dynamic and plastic changes underlying fear, extinction, and reinstatement. Manipulations targeting these different types of plasticity could constitute a therapeutic tool for the treatment of anxiety disorders.

Neuropsychopharmacology (201 I) 36, 2276-2285; doi:I0.1038/npp.20 II.II5; published online I3 July 20 I I
\end{abstract}

Keywords: fear; extinction; reinstatement; mPFC; BLA

\section{INTRODUCTION}

Auditory fear conditioning (FC) develops when an initially neutral tone is paired with a foot-shock as unconditioned stimulus (US). As a result, the tone becomes a conditioned stimulus (CS), which is capable of evoking conditioned fear responses, such as conditioned freezing behavior. However, continuous presentations of the CS without the US lead to progressive suppression of conditioned fear responses, a phenomenon known as fear extinction. Converging lines of evidence, based on electrophysiological approaches, support the involvement of the medial prefrontal cortex (mPFC) and basolateral amygdala (BLA) in FC and extinction. For example, increased neural activity in the prelimbic area of the mPFC (PL) was observed in rats in response to CS presentation (fear excitation; Burgos-Robles et al, 2009). In contrast, recall of extinction memory (longterm fear inhibition) was associated with increased neural activity in the infralimbic mPFC (IL; Milad and Quirk, 2002) as well as increased synaptic efficacy in mPFC inputs, from the mediodorsal thalamus (Herry and Garcia, 2002) and the hippocampus (Garcia et al, 2008). Similarly, enhanced long-term potentiation (LTP) in the BLA was associated

*Correspondence: Dr M Maroun, Department of Neurobiology, Faculty of Natural Sciences, University of Haifa, Haifa 31905, Israel, Tel: + 009724824 9657, Fax: + 0097248288 108,

E-mail: mmaroun@psy.haifa.ac.il or mouna.maroun@gmail.com

Received 7 February 2011; revised 25 May 201 I; accepted 25 May 2011 with fear learning (eg, LeDoux, 2000). Potentiation of auditory-evoked potentials in the BLA following FC was also reported (Rogan et al, 1997; Garcia et al, 1998; Tang et al, 2001). Furthermore, it was found that repetitive presentations of the tone CS induced not only extinction of conditioned fear responses, but also suppression of the potentiation of auditory-evoked potentials (Tang et al, 2001). Together, these studies suggest a dynamic interaction between the MPFC and BLA during both fear excitation and inhibition processes (Akirav et al, 2006; Akirav and Maroun, 2007). More precisely, the MPFC (both PL and IL areas) may integrate inputs from various areas, including the BLA, to gate the expression of fear via projections to inhibitory or excitatory circuits within the amygdala (Sotres-Bayon and Quirk, 2010). To our knowledge, little is known about the way by which the MPFC and the BLA interact in fear and extinction.

To explore this issue, we examined in behaving rats changes in the evoked field potentials (EFPs) in these reciprocal connections, mPFC-BLA and BLA-mPFC pathways, following auditory FC and extinction.

\section{SUBJECTS AND METHODS}

\section{Subjects and Surgery}

The experiments were performed using male SpragueDawley rats (Harlan Laboratories, Jerusalem) weighing 200-280 g. Animals were housed in Plexiglas cages (five 
rats per cage) and were maintained on a free-feeding regimen with a 12-h light-12-h dark schedule. A week after arrival, the rats were anesthetized with $4.8 \mathrm{ml} / \mathrm{kg}$ Equithesin $\left(2.12 \% \mathrm{w} / \mathrm{v} \mathrm{MgSO}_{4}, 10 \% \mathrm{v} / \mathrm{v}\right.$ ethanol, $39.1 \% \mathrm{v} / \mathrm{v}$ propylene glycol, $0.98 \% \mathrm{w} / \mathrm{v}$ sodium pentobarbital, and $4.2 \% \mathrm{w} / \mathrm{v}$ chloral hydrate), and placed in a stereotaxic frame, with body temperature maintained at $37 \pm 0.5^{\circ} \mathrm{C}$. Rats were surgically implanted with stimulating and recording electrodes made of two intertwined stainless steel wires $(80 \mu \mathrm{m}$ in diameter) insulated except at the tip. The procedures were performed in strict accordance with University of Haifa regulations and National Institute of Health guidelines (NIH publication number 8023). The electrodes were aimed to the $\mathrm{mPFC}$ (anteroposterior, $+3 \mathrm{~mm}$ relative to bregma; lateral, $\pm 0.5 \mathrm{~mm}$; ventral, $4-5 \mathrm{~mm}$ ), and the BLA (anteroposterior, $-2.8 \mathrm{~mm}$ relative to bregma; lateral, $\pm 5.0 \mathrm{~mm}$; ventral, $7.6 \mathrm{~mm}$ ).

After the final placement of the electrodes, they were fixed in place with dental cement. Following the implantation, the rats were randomly housed two per cage and allowed to recover in the animal room for at least 7 days before recording sessions.

Both electrodes were implanted in the same hemisphere; however, some animals were implanted on the right hemisphere and others on the left, in order to avoid effects of laterality.

\section{Electrophysiology}

Stimulating and recording procedures. After recovering from surgery, the animals were habituated for 5-7 days to transportation and to being connected and disconnected from a miniature head-stage. The miniature head-stage was connected to a flexible cable that was relayed through the top of the recording chamber (a Plexiglas box: $50 \times 30 \times$ $30 \mathrm{~cm}$ ) to a multichannel commutator. This system allowed the animal to move freely within the chamber while recording electrophysiological data.

Field potentials in the BLA or MPFC were evoked by single $0.1 \mathrm{~ms}$ rectangular monophasic pulses that were delivered to either the $\mathrm{MPFC}$ or the BLA, respectively. These pulses were amplified (gain $\times 1000$; band-pass: $0.001-1 \mathrm{kHz}$ ) by an AM-systems amplifier, displayed on an oscilloscope, digitized at $10 \mathrm{kHz}$ (CED) and stored to disk for off-line analysis (Spike-2 software).

For each recording session, 20 responses were elicited at a stimulus frequency of $0.1 \mathrm{~Hz}$. Each average response was established using 20 responses from the same recording session. Stimulation intensity throughout the experiment for each rat was chosen (from the baseline input-output curves: $0.1-0.5 \mathrm{~mA}$ ) according to the stimulation intensity that produced a response representing approximately $50 \%$ of the maximal response.

To determine whether the field potentials evoked in mPFC and BLA are synaptic responses or generated by cell firing in these structures, extracellular single-unit activity was recorded in rats ( $n=3$ per pathway) acutely prepared under anesthesia. Stimulation of either BLA or mPFC was performed as described above, but with low stimulus intensities. Evoked responses in either BLA or mPFC were recorded using glass microelectrodes filled with $2 \mathrm{M}-\mathrm{NaCl}$, having tip diameters of $5-10 \mu \mathrm{m}$ and DC resistances in saline between 1 and $5 \mathrm{M} \Omega$. The responses were filtered and amplified (band-pass: $0.3-10 \mathrm{kHz}$, gain: 10000 for singleunit activity). Recording placements were determined by the response pattern of the recorded field potential.

\section{Behavior}

Conditioning, extinction, and reinstatement. Fear conditioning: FC took place in a conditioning chamber with a grid floor and transparent Plexiglas walls. The CS was a $30 \mathrm{~s}$ tone $(4 \mathrm{kHz} ; 80 \mathrm{~dB}$ sound pressure level) and the US was a $0.8 \mathrm{~mA}, 1 \mathrm{~s}$ foot-shock delivered through the grid floor, with the CS and US co-terminating. The inter-trial interval was around $2 \mathrm{~min}$ throughout. Animals received three tones paired with foot-shocks. The freezing response of each rat to the first tone, before initial presentation of the first shock, was considered as baseline response to the tone (pre). The responses of the rat to the next two tones were considered conditioning responses (T2-3). Following FC, the animals were taken back to their home cage.

Extinction: In order to condition the rats specifically to the tone and minimize the effect of context, rats were habituated for 3 days before conditioning ( $20 \mathrm{~min}$ per day) to a safe box that was of circular shape with transparent Plexiglas walls and a black Plexiglas floor.

At $24 \mathrm{~h}$ following conditioning, rats started with extinction training, which consisted of 20 min per day in the safe box during which 10 tones were delivered in the absence of foot-shocks.

\section{Reinstatement of FC}

Fear was reinstated $24 \mathrm{~h}$ after the completion of the last extinction training and immediately after post-Ext3 EFPs recording. Specifically, animals were placed in the chamber where extinction took place and after 2-min adaptation period, they were exposed to an unsignaled single shock reminder (eg, Storsve et al, 2010; one electrical shock at $0.8 \mathrm{~mA}$ for $0.5 \mathrm{~s}$ ). Animals were returned to their home cages $1 \mathrm{~min}$ following the delivery of the reinstating stimulus and $24 \mathrm{~h}$ later, they were brought back to the chamber and freezing levels were measured in response to the presentation of three tones.

Between each session, the boxes were thoroughly cleaned with odorous clean wipes.

The behavior of the rat was observed by means of a video camera monitoring system (for further details, see Hikind and Maroun, 2008).

\section{Groups}

Fear conditioning ( $F C$ only, $\mathrm{n}=19$ ). On the FC day, animals in the Extinction and FC groups underwent training to associate between the tone and the foot-shock (see above).

Rats in the 'FC only' group underwent electrophysiological recording in the absence of extinction training and they were returned to their home cage immediately after each recording session.

Extinction $(F C+$ Extinction, $\mathrm{n}=22)$. Rats in the ' $\mathrm{FC}+$ Extinction' group underwent conditioning and extinction training over 3 days (Ext1, Ext2, and Ext3). 
Control $(\mathrm{n}=17)$. Animals in the 'Control' group were placed in the conditioning chamber for the same total period as the 'FC + Extinction' group and were exposed to the same number of tones on the FC day in the absence of foot-shocks and to the same number of tones on the extinction days.

Reinstatement of fear $(\mathrm{n}=19)$. Reinstatement of fear was tested in an additional and different set of animals, which underwent fear, extinction of fear, and electrophysiological recording protocols as reported above. Following the termination of the last electrophysiological session (post Ext3), reinstatement took place in the context of extinction (eg, Storsve et al, 2010). Following a 2-min adaptation period, rats were exposed to a single foot-shock $(0.8 \mathrm{~mA}$ for $0.5 \mathrm{~s}$ ) and animals were returned to their home cages $1 \mathrm{~min}$ following the delivery of the reinstating stimulus. After $24 \mathrm{~h}$, fear responses to the presentation of three tones and EFPs changes were monitored.

\section{Electrophysiological Recording}

As we were interested in the long-term effects of FC and extinction on EFPs, electrophysiological recording took place $24 \mathrm{~h}$ following FC (post FC), $24 \mathrm{~h}$ following the first and the last extinction training sessions (post Ext1 and post Ext3, respectively) and $24 \mathrm{~h}$ following reinstatement.

Histology. Electrode placements (recording and stimulating electrodes) were verified in all the rats. After the last session of electrophysiological testing, rats were deeply anesthetized with an overdose of Equithesin and marking lesions were made by passing anodal currents ( $10 \mathrm{~mA}$ for $3 \mathrm{~s}$ ) through the metal electrodes.

Electrode tips were examined under a light microscope following Nissl staining. Figure 1 shows a schematic drawing of the BLA (coronal view at position -3.14 and $-3.30 \mathrm{~mm}$ posterior to bregma) and $\mathrm{mPFC}$ (coronal view at position +3.20 and $+2.70 \mathrm{~mm}$ anterior to bregma) electrode placements. Solid black circles indicate the locations. We excluded both behavioral and electrophysiological data from rats in which either electrode was imprecisely located (total six rats).
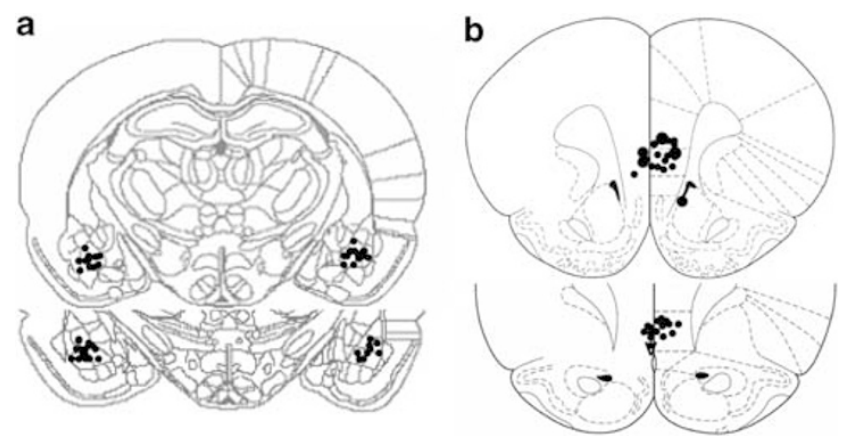

Figure I Schematic drawing indicating electrode tip positions within the medial prefrontal cortex (mPFC) and the basolateral amygdala (BLA). Shown is a coronal view of (a) the MPFC and (b) the BLA at position $3.20 \mathrm{~mm}$ and $2.70 \mathrm{~mm}$ anterior to the bregma, and at $-3.14 \mathrm{~mm}$ and $-3.30 \mathrm{~mm}$ posterior to the bregma, respectively. Adapted with permission from Elsevier 1986, Paxinos and Watson, 1986.
Data analysis. Behavior: Freezing results are presented as the percentage of time spent in freezing during each presentation of the 30-s tone. The results for the 10 tones delivered during extinction training are presented as five blocks, each representing the average of two trials.

In the reinstatement experiment, $24 \mathrm{~h}$ post reinstatement animals were presented with three tones that were averaged.

Electrophysiology: The amplitudes $\mathrm{A}$ and $\mathrm{A}^{\prime}$ of the field potentials (measured from the top peak (P1 or $\mathrm{P}^{\prime}$ ) to the bottom of the sink of the negative wave $\left(\mathrm{N} 1\right.$ or $\left.\mathrm{N1}^{\prime}\right)$; Figure 3 ) were expressed as the mean percentage ( \pm SEM).

The amplitudes were normalized to the individual baseline values (measured over 3 days) of animals for each group and were expressed as percentage of change from baseline which was set on $100 \%$.

Differences among the groups were determined by independent $t$-tests or analysis of variance (ANOVA). All post hoc comparisons were made by the least significant difference multiple-comparison test (LSD). Furthermore, Pearson's correlational test was used to examine correlations between the EFPs and the relevant freezing responses.

\section{RESULTS}

\section{Histology}

Histology showed that recording and stimulating electrodes aimed to the mPFC were mainly located at the upper part of the IL with some rats having their electrodes close to the border with the PL area. As behavioral and electrophysiological data of the animals did not differ, these rats were pooled together and therefore we preferentially use the term 'mPFC'. Furthermore, electrodes were implanted in the same side for each animal but some animals had their electrodes on the left side and others on the right side. There was no effect (behavior or electrophysiology) of the side of implantation (data not shown).

The two pathways were investigated in different animals and the distribution of animals according to the two pathways was as following: For the descending mPFC-BLA pathway (Control, $n=10$; FC only, $n=7$; FC + Extinction, $n=11$ ), and for the ascending BLA-mPFC pathway (Control, $n=7$; FC only, $n=12$; FC + Extinction, $n=11$ ).

\section{Behavior}

Behavioral measurements for animals implanted in both pathways were not statistically different; therefore, data of both pathways were pooled for analyses. FC (FC only, $n=19$ ), Extinction (FC + Extinction, $n=22$ ), and Control (Control, $n=17$ ).

To verify that initial freezing levels were similar, we compared the baseline freezing levels at the first tone (before conditioning). Freezing to the first tone were very low and no differences were observed between the groups at first tone, suggesting comparable freezing levels and active exploration $(\mathrm{F}<1$; Figure 2$)$. In contrast, FC significantly enhanced freezing levels in T2-3 in the conditioned animals as compared with controls $(\mathrm{F}(2,55)=62.3 ; p<0.0001$; Figure 2). Post hoc analysis did not show significant differences between the conditioned groups (FC only and $\mathrm{FC}+$ Extinction). 


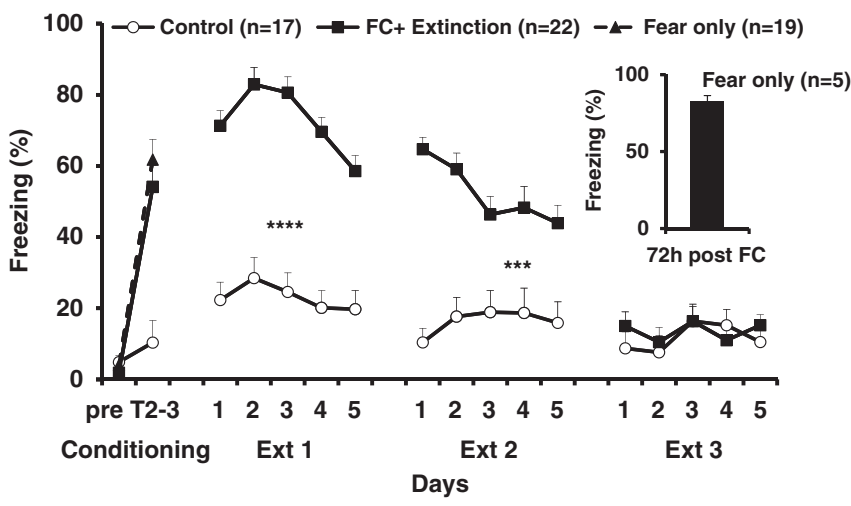

Figure 2 Freezing levels following FC and subsequent extinction. The 'FC only' and 'FC + Extinction' groups were trained to associate between tone and foot-shock on the FC day. At $24 \mathrm{~h}$ following conditioning, the 'FC + Extinction' group underwent extinction training for three consecutive days (ExtI, Ext2, and Ext3). The 'Control' group underwent the same protocol as the 'FC + Extinction' group but in the absence of foot-shocks. The 'FC + Extinction' showed high freezing levels (calculated as the percentage of time the rats spent freezing during the 30-s tone) on Ext I $(* * * * p<0.00$ I) and Ext2 (*** $p<0.005)$, indicating robust acquisition of the association of tone-shock memory. On Ext3, the freezing levels of the 'FC + Extinction' group were not significantly different from the levels of the 'Control' group, and thus indicating the success of extinction training. Inset: a subset of the rats that underwent FC had their long-term fear memory tested by re-exposure to the tone 3 days later. These rats show robust retention of fear memory.

Following fear learning, the 'FC' group was divided in two similar sub-groups: the 'FC only' group (FC only, did not undergo further behavioral testing) and the 'Extinction' group (FC + Extinction). There was no significant difference between the two groups in the freezing levels during FC (data not shown).

ANOVA with repeated measures on the three extinction days showed a significant effect of group $(F(1,37)=18.53$; $p<0.001)$, a significant effect of day $(\mathrm{F}(2,74)=88.4$, $p<0.001)$, and a significant interaction between group and day $(\mathrm{F}(2,74)=21.52, p<0.01)$. ANOVA performed on the freezing levels of the 'Control' and the 'FC+Extinction' groups on each of the extinction days showed significant differences between the two groups during Ext1 $(\mathrm{F}(1,37)=$ 40.4; $p<0.001)$ and Ext2 $(\mathrm{F}(1,37)=4.8 ; p=0.037)$. In contrast, no significant difference between the two groups were found on the last day of extinction (Ext3 $\mathrm{F}<1$; NS), suggesting that the 'FC + Extinction' group extinguished the fear response and expressed similar freezing levels as those of the 'Control' group.

In order to verify that the FC protocol used in this experiment is robust and able to produce long-lasting fear memory, a subset of animals underwent conditioning and was tested $72 \mathrm{~h}$ after FC. The results showed high freezing level (75.07 $\pm 7.4 \%$; Figure 2, inset) suggesting that the conditioning protocol was effective in producing long-term fear memory that could last over $72 \mathrm{~h}$.

\section{Electrophysiology}

EFPs in the MPFC-BLA circuit. As previously reported, stimulation of mPFC elicited a negative-going EFPs in the BLA (Maroun, 2006). Simultaneous studies of multi-unit activity and EFPs showed that the latency of the evoked unit activity coincided with peak latency of the negative component of the EFP (Figures $3 \mathrm{a}$ and $\mathrm{b}$ ). This suggests that the EFPs arise from combined activity of neurons distributed in the BLA.

Stimulation of the BLA also elicited a negative-going EFP in the MPFC (Maroun and Richter-Levin, 2003). The shape, amplitude, and latency of the EFPs were highly reproducible and corresponded to neuronal activity (Figures $3 c$ and $d$ ).

Thus, in both pathways the latency of the evoked unit activity coinciding with peak latency of the negative component of the EFP, suggested that the components measured in this study reflect neuronal firing.

MPFC-BLA pathway: learning-induced changes in EFPs. ANOVA with repeated measures $(3 \times 3$; three groups $\times 3$ baseline days) did not reveal significant differences between the groups $(\mathrm{F}(2,25)=0.893$; NS; Figure $4 \mathrm{a})$ or the baseline measurement day $(\mathrm{F}(1,25)=0.16 ; \mathrm{NS})$ or of interaction between the groups and the baseline day $(\mathrm{F}(2,25)=$ 1.2 ; NS).

ANOVA on the EFPs amplitudes $24 \mathrm{~h}$ following FC (post FC) revealed significant differences between the three groups $(\mathrm{F}(2,25)=5.9 ; p<0.001)$. Post hoc analysis showed that the 'FC only' and 'FC + Extinction' groups exhibited a significant increase in EFPs amplitudes (115.3 $\pm 2.5 \%$; $p<0.01$ and $113.09 \pm 3.4 \%$, respectively; $p<0.05$ different from Control: $101.3 \pm 2.6 \%)$.

Changes in the EFPs $24 \mathrm{~h}$ following conditioning (post FC) positively correlated with freezing levels during the FC session $(r=0.54 ; p<0.01)$ suggesting that an increase in the freezing level is associated with an increase in the EFPs.

ANOVA with repeated measures on the EFPs amplitudes on the 2 extinction days revealed significant differences between the groups $(\mathrm{F}(2,25)=17.5 ; p<0.001)$, without significant effect of day $(\mathrm{F}(1,25)=0.79$; NS) or of the interaction $(\mathrm{F}(2,25)=2.4$; NS).

Further statistical analysis performed on the EFPs amplitudes across the extinction days revealed significant differences between the groups in post Ext1 $(\mathrm{F}(2,25)=$ $13.43 ; p<0.005)$ and post $\operatorname{Ext} 3(\mathrm{~F}(2,25)=13.48 ; p<0.001)$. Post hoc analysis on post Extl revealed that the 'FC+ Extinction' group had significant depression of EFPs amplitudes $(86.17 \pm 4.6 \%)$ compared with both 'FC only' group, which maintained the increase in the EFPs amplitudes $(122.18 \pm 5.7 \% ; p<0.005)$, and 'Control' group (102.42 \pm $3.46 \% ; p<0.05)$. Furthermore, intensive extinction training over 3 days (post Ext3) caused a further depression in the EFPs amplitudes in the 'FC + Extinction' group (75.9+ $4.88 \%)$ compared with the 'FC only' (119.9 $\pm 7.6 \%$; $p<0.0001)$ and the 'Control' groups $(98.7 \pm 2.2 \% ; p<0.005)$.

No correlations were found between the freezing levels and changes in EFPs at the corresponding days.

Overall, these results show that FC and extinction are associated with an increase and decrease in the EFPs amplitudes, respectively.

BLA-mPFC pathway: learning-induced changes in EFPs. ANOVA with repeated measures did not reveal any significant difference between the three groups during the baseline days $(\mathrm{F}(2,27)=0.78$; NS; Figure $4 \mathrm{~b})$. The percentage change in the EFPs amplitude $24 \mathrm{~h}$ after fear (post FC) 

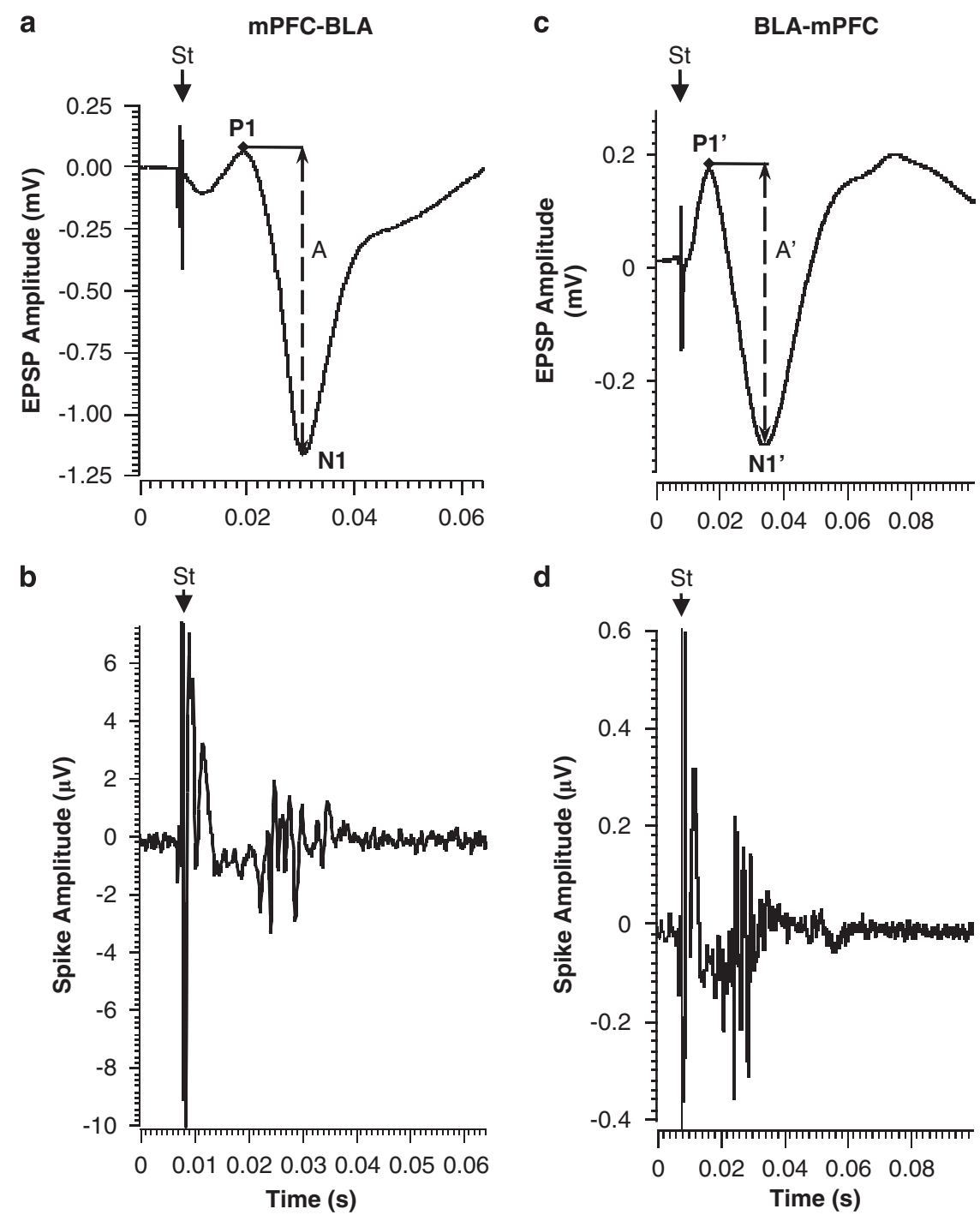

Figure 3 Unit responses and corresponding EFPs. Examples of field potential (a, c) and unit activity (b, d) evoked in the mPFC-BLA (left) and BLA-mPFC (right) pathways. Latency of evoked single-unit activity coincided with peak latency of the negative component of the fields potentials: 24 ms for the mPFC-BLA and 26 ms for BLA-mPFC pathway. St, stimulus artifact. The amplitudes A and $\mathrm{A}^{\prime}$ of the field potentials (measured from the top peak (PI or PI') to the bottom of the sink of the negative wave (NI or $\mathrm{NI}^{\prime}$ ).

did not significantly differ between the three groups $(\mathrm{F}(2,27)=1.7 ; \mathrm{NS})$, indicating that $\mathrm{FC}$ did not induce persistent changes in the EFPs amplitudes.

However, changes in the EFPs $24 \mathrm{~h}$ following conditioning (post FC) negatively correlated with freezing levels during the FC session $(r=-0.48 ; p<0.05)$.

ANOVA with repeated measures on the percentage change in the amplitudes of EFPs following the extinction days (post Ext1 and Ext3) showed significant differences between the three groups $(\mathrm{F}(2,27)=7.17 ; p<0.001)$.

One-way ANOVA on the amplitudes of EFPs at post Ext1 showed a significant difference between the groups $(\mathrm{F}(2,27)=4.85 ; p<0.05)$. Post hoc analysis revealed that the 'FC + Extinction' group had a significant increase in the amplitude of EFPs compared with the 'FC only' group (110.46 $\pm 4.85 \%$ vs $93.10 \pm 3.126 \%$; $p<0.005)$. The average EFPs amplitudes of the 'Control' group lay between those of the other two groups and did not differ significantly from either of them $(103.8 \pm 4.32 \% ; \mathrm{F}<1)$.
Similarly, the percentage change in EFPs amplitudes significantly differed between the groups on post Ext3 $(\mathrm{F}(2,27)=11.91 ; p<0.005))$. Post hoc analysis showed that the 'FC + Extinction' group differed from the 'FC only' group and showed a significant potentiation of the amplitudes of EFPs $(120.21 \pm 4.7 \%$ and $90.23 \pm 4.51 \%$, respectively; $p<0.005$ ), and from the 'Control' group $(99.94 \pm 4.3 \%, p<0.01)$. These results indicate that extinction of FC-induced potentiation of EFPs amplitudes in the BLA-mPFC pathway.

Reinstatement of fear is associated with recovery of fear responses and reversal of EFPs in both pathways. From the above experiments, it is clear that fear and extinction are mediated by differential and opposite changes in EFPs amplitudes. Next, we hypothesized that if the circuit of mPFC-BLA is indeed plastic then reinstatement of fear should be associated with reversal of the changes that were observed following fear extinction. In order to test this 


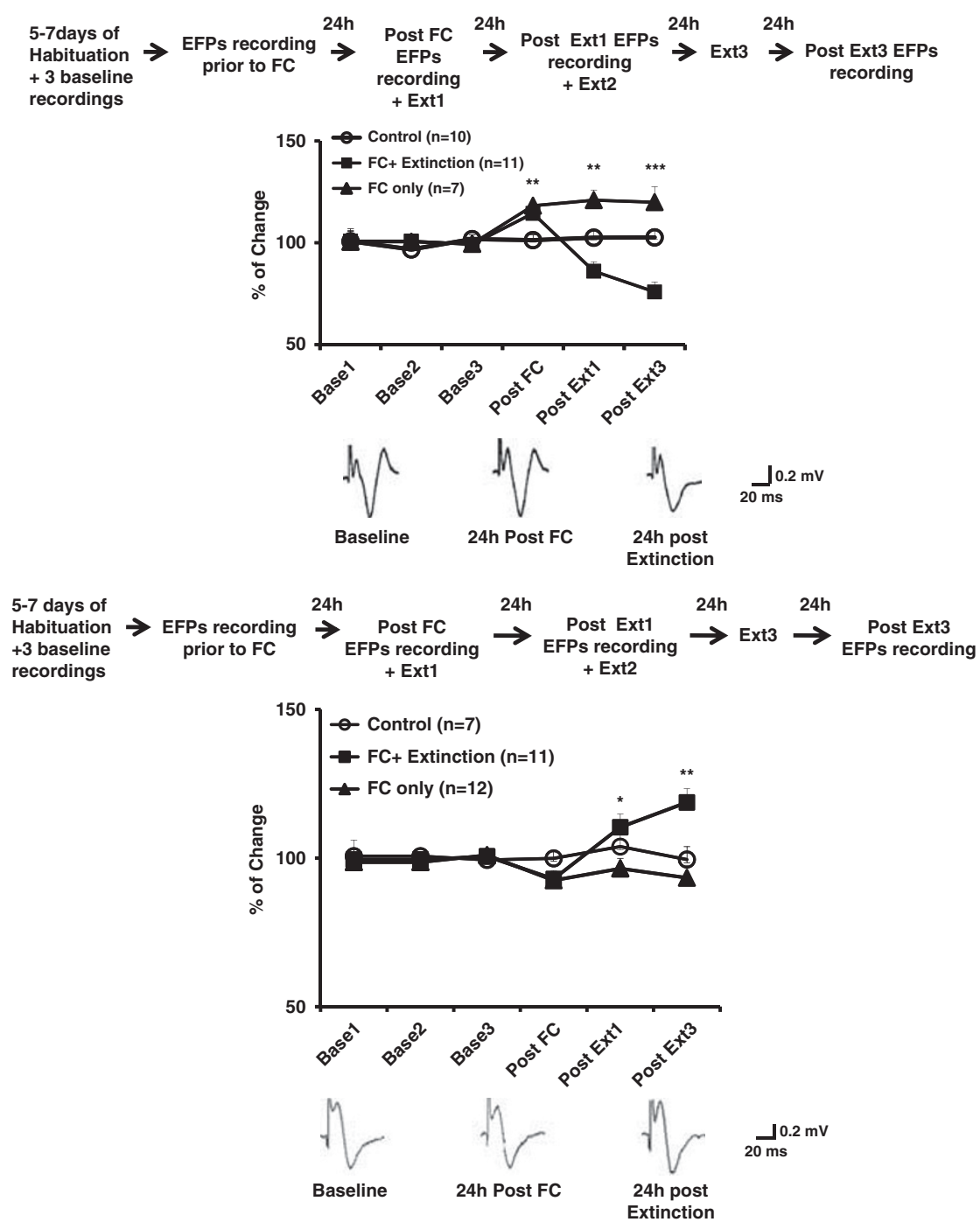

Figure 4 (a) Learning-induced changes in the MPFC-BLA pathway. Top: changes in the EFPs amplitude are expressed as a percentage of change from the baseline recording that was averaged for the two recording days. The EFPs amplitudes $24 \mathrm{~h}$ after FC (post FC) were significantly different between the three groups. Post hoc analysis showed a significant increase in EFPs amplitudes for the 'FC only' and 'FC + Extinction' groups compared with 'Control' group, whose EFPs amplitudes did not significantly differ from the baseline levels. By contrast, extinction training was concomitant with a reduction in EFPs amplitudes in the 'FC + Extinction' group compared with the 'FC only' and 'Control' groups post Extl and Ext3 (post Extl, *** $<<0.005)$ and post Ext3, $* * * p<0.00$ I). Bottom: representative traces of the main effects. (b) Learning-induced changes in the BLA-mPFC pathway. Top: FC was not associated with significant changes in EFPs amplitudes $24 \mathrm{~h}$ after conditioning. The amplitudes of EFPs were significantly different between the groups on post Ext I $(* p<0.05)$ and post Ext3 (** $p<0.005)$. Extinction training was accompanied with a significant potentiation of EFPs in the 'FC + Extinction' group compared with the 'FC only' and 'Control groups' (* $p<0.005)$. Bottom: representative traces of the main effects.

hypothesis, after the completion of the last recording day ( $24 \mathrm{~h}$ post Ext3), the rats were randomly assigned to either Reinstatement or No Reinstatement. Fear was reinstated in the extinction box by applying an unsignaled electrical shock (eg, Storsve et al, 2010). At $24 \mathrm{~h}$ after the reinstatement, freezing levels to the presentation of three tones and changes in the EFPs were measured.

\section{Behavior}

Analysis of the results and inspection on Figure 4a show that the freezing levels of the present experiment did not differ from those of the group of FC + Extinction in the previous experiment at any time point $(\mathrm{F}<1$; Figure $5 \mathrm{a})$.
In contrast, the reinstatement of fear was associated with recovery of the freezing levels measured $24 \mathrm{~h}$ following the protocol of reinstatement as the group that underwent reinstatement significantly differed from the group, which did not undergo reinstatement of fear (Reinstatement, $n=11$, No Reinstatement, $n=9 ; \mathrm{t}(17)=24.8 ; p<0.0001$; Figure 5a).

mPFC-BLA pathway: effects of reinstatement of fear. Before reinstatement/no reinstatement of fear, no significant differences were observed in the changes in EFPs in the present experiment as compared with the previous experiment $(\mathrm{F}<1$; Figure $5 \mathrm{~b})$. Following $\mathrm{FC}$ there was a significant enhancement in the amplitude of the EFPs compared with baseline $(119.5 \pm 2.3 \% ; \mathrm{t}(8)=8.9 ; p<0.0001$ for difference 


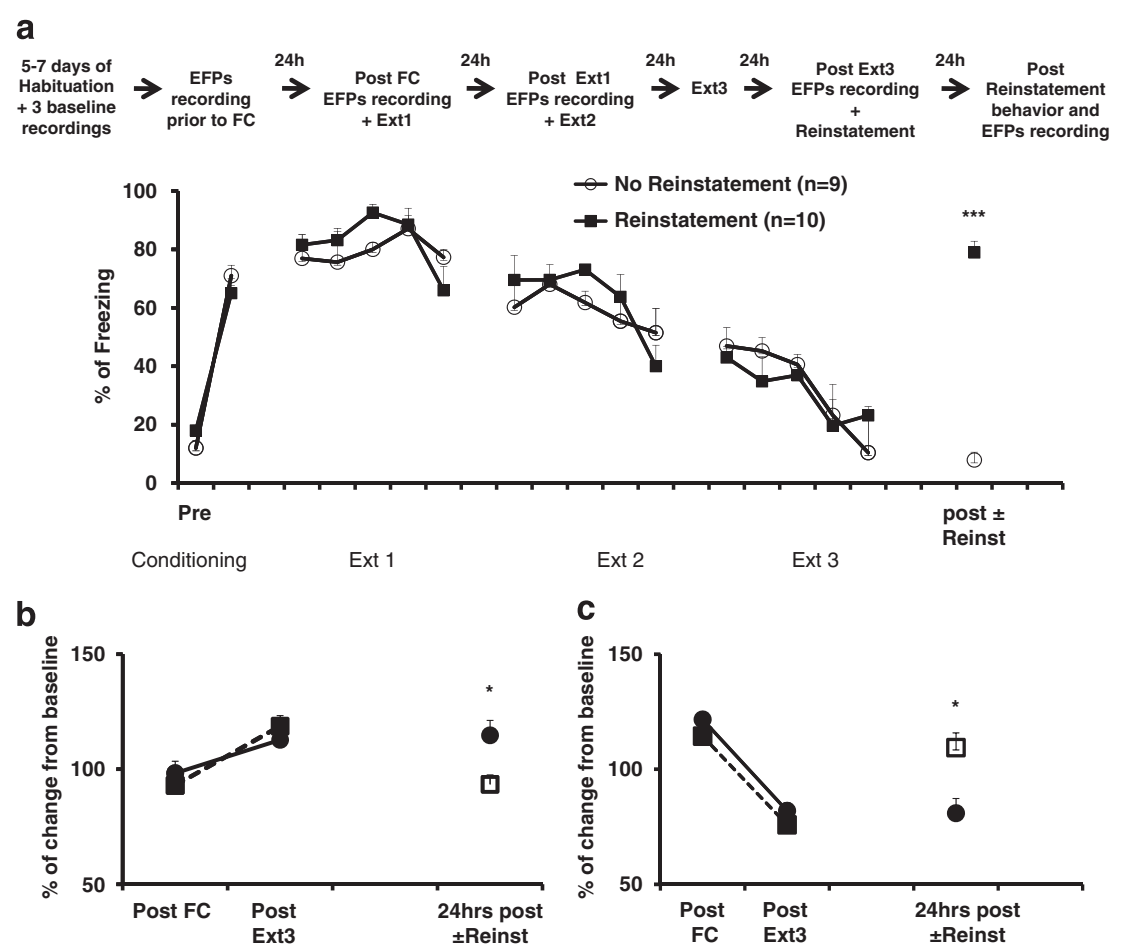

Figure 5 Reinstatement of fear reverses changes following extinction. At $24 \mathrm{~h}$ following the last extinction training and after the recording of post Ext3 EFPs, fear was reinstated by placing the animals in the conditioning box in which they either received an unsignaled shock (Reinstatement) or did not receive any shock (No Reinstatement). At $24 \mathrm{~h}$ after reinstatement freezing values and EFPs recording were measured. (a) Animals were fear conditioned and for underwent extinction training for 3 days. At $24 \mathrm{~h}$ after the last extinction session they were assigned to either No Reinstatement or Reinstatement groups. The results show that the freezing levels of the animals in this experiment (black circles) were not different from those in the previous experiment (dotted line) at any time point. Reinstatement of fear resulted in recovery of the fear responses in the group that received the unsignaled shock compared with the group, which did not undergo reinstatement of fear as $24 \mathrm{~h}$ following reinstatement the two groups significantly differ (**** $p<0.000 \mathrm{I}$ ). (b) The mPFC-BLA pathway: EFPs were normalized to the values that were reordered during baseline recordings. Similar to the results observed in Figure 4a, FC and extinction of fear were associated with potentiation/depression, respectively. Furthermore, fear was associated with repotentiation of the EFPs compared with baseline values (Reinstatement group: $n=5$, No Reinstatement group, $n=4 ; *=0.01$ ). The dotted line represents the data of Figure 3 a to show comparable values in the two experiments. (c) The BLA-mPFC pathway: EFPs were normalized to the values that were reordered during baseline recordings. Extinction of fear was associated with potentiation of the EFPs amplitudes and reinstating of fear was associated with depotentiation of the EFPs values as compared with those that were recorded post extinction training (Reinstatement group: $n=6$, No Reinstatement group, $n=4$; ${ }^{*} p=0.0 \mathrm{I}$ ). The dotted line represents the data of Figure $4 b$ to show comparable values in the two experiments.

from $100 \%)$. Similarly, extinction training was also associated with a significant reduction in the EFPs amplitude $(81.6 \pm$ $1.8 \% ; \mathrm{t}(8)=9.2 ; p<0.0001$ for difference from $100 \%)$.

Reinstatement of FC was associated with re-potentiation of the EFPs in the Reinstatement group compared with the group, which did not undergo reinstatement (Reinstatement, $n=5$ : $\quad 107.4 \pm 6.2 \%$; No Reinstatement, $n=4$ : $84.9 \pm 6.4 \%: \mathrm{t}(7)=3.26 ; p<0.001$; Figure $5 \mathrm{~b}$ ).

Furthermore, correlations calculated between the freezing scores $24 \mathrm{~h}$ post reinstatement and the changes observed in the EFPs amplitudes show positive correlation between the two variable $(r=0.925, n=9 ; p<0.001)$, suggesting that an increase in the freezing level is associated with an increase in the EFPs.

BLA-mPFC pathway: effects of reinstatement of fear. Before reinstatement/no reinstatement of fear, no significant differences were observed in the changes in EFPs in the present experiment as compared with the previous experiment $(\mathrm{F}<1$; Figure $5 \mathrm{c}$ ). Only after extinction training (post Ext3), animals showed a significant potentiation in the EFPs amplitude compared with baseline recordings (118.3 $\pm 3.8 \%$; $\mathrm{t}(9)=4.7 ; p<0.001$ for difference from $100 \%$; Figure $5 \mathrm{c}$ ).
Reinstatement of FC was associated with depression of the EFPs in the Reinstatement group compared with the group, which did not undergo reinstatement (Reinstatement, $n=6: 93.4 \pm 3.2 \%$ : No Reinstatement, $n=4: 114.79 \pm$ $7.4 \% ; \mathrm{t}(8)=4.88 ; p<0.001$; Figure $5 \mathrm{c}$ ).

Furthermore, correlations calculated between the freezing scores $24 \mathrm{~h}$ post reinstatement and the changes observed in the EFPs amplitudes following the reinstatement show negative correlation between the two variable $(r=-0.833$, $n=10 ; p<0.003)$, suggesting that as the levels of freezing increase, the potentiation is reduced.

\section{DISCUSSION}

We examined learning-induced changes in EFPs amplitudes in the mPFC-BLA circuit in the freely moving rat following auditory FC, extinction, and reinstatement of fear. Our underlying hypothesis was that because the MPFC and the BLA are differentially involved in fear and extinction (eg, Akirav and Maroun, 2007), then their opposite roles should be expressed through differential synaptic changes in the EFPs. We provide here an evidence for the plastic nature of 
the mPFC-BLA circuit following learning. Precisely, we clearly show that fear, extinction, and reinstatement of fear generate differential and opposite changes in the mPFCBLA circuit that are specific to the learning experience.

\section{FC in the mPFC-BLA Circuit}

FC was associated with an increase in the EFPs amplitudes in the mPFC-BLA pathway up to $72 \mathrm{~h}$ post conditioning. Such a potentiation is consistent with previous reports showing that the BLA is the locus for fear memory (Brambilla et al, 1997; Rogan et al, 1997; Fanselow and LeDoux, 1999; Maren, 1999; LeDoux, 2000; McKernan and Shinnick-Gallagher, 1997; Vouimba et al, 2004; Rodríguez Manzanares et al, 2005; Kavushansky et al, 2006).

In contrast to previously reported data, FC did not induce significant changes in the reciprocal BLA-mPFC pathway (Garcia et al, 1999; Herry and Garcia, 2002; Santini et al, 2008). One explanation for this inconsistency could be that in this study, we used a mild conditioning protocol, which is not sufficient to induce lasting changes in the BLA-mPFC pathway. Such a hypothesis is consistent with recent data showing that a conditioning protocol similar to the one used in this study did not affect LTP magnitude in the hippocampal CA1 (Motanis and Maroun, 2010). This suggests that the BLA might be more susceptible to this specific conditioning protocol compared with the mPFC and the CA1.

\section{Extinction in the mPFC-BLA Circuit}

In the mPFC-BLA pathway, extinction training resulted in a significant and long-lasting depression of EFPs amplitudes, suggesting a shift from LTP-like to LTD-like following extinction training (low freezing levels, ie, no danger). These results confirm our previous report that under normal conditions, low-frequency stimulation inducedLTD in the mPFC-BLA is favored, but under stressful conditions, LTD is inhibited (Maroun, 2006). Similarly, Rogan et al (2005) have suggested that learned safety involves an LTD-like mechanism and that the same lowfrequency stimulation that induces LTD in the amygdala in vitro attenuate conditioned fear responses in vivo (Lin et al, 2003).

In the BLA-mPFC pathway, a significant increase in the amplitude of EFPs was observed following extinction training, indicating that extinction might be achieved via potentiation of the BLA-mPFC pathway. This observation is consistent with data showing that LTP in the MPFC was associated with the maintenance of extinction while LTD was associated with the return of fear (Herry and Garcia, 2002). Moreover, we have previously showed that exposure to a stressful experience inhibits the induction of LTP in the mPFC (Maroun and Richter-Levin, 2003; Rocher et al, 2004; Richter-Levin and Maroun, 2010) and impairs extinction of FC (Akirav and Maroun, 2007; Izquierdo et al, 2006; Akirav et al, 2009). Conversely, electrical stimulation of the infralimbic cortex reduced freezing to conditioned tones (Milad and Quirk, 2002), probably by reducing the responsiveness of the medial section of the central amygdala's output neurons to synaptic activation from the BLA (Royer and Paré, 2002; Quirk et al, 2003).
Some reports suggest that within the $\mathrm{mPFC}$ the PL and IL have opposite roles in fear and extinction. On the one hand, while the IL has a crucial role in inhibiting fear and promoting extinction, the PL on the other hand has a role in exciting fear expression (Vidal-Gonzalez et al, 2006).

In our study, the majority of the tip of the stimulating electrodes was located in the upper IL and fewer had the stimulating electrode tip located in the borders between the PL and the IL. As IL stimulation could also lead to activation of the PL sub-region, we cannot exclude that the effects observed in this study, at least in part, are mediated by joint stimulation or interaction between the IL and the PL. Yet, we did not observe significant differences between animals that had their stimulating electrode in the IL and those who had it adjacent to the PL. Furthermore, rats that had their tip of electrodes in the PL did not show any difference in the kinetic of changes in EFPs amplitudes during the different stages of fear and extinction as compared with rats implanted in the IL.

It should be noted that similar expression of Fos was reported in the IL and the PL following fear extinction in a recent study by Kim et al (2010). Furthermore, consistent with the present findings, Judo et al (2010) and Koseki et al (2009) showed that synaptic transmission in the PL was reduced during the first extinction trial (high freezing), but was potentiated following extinction retrieval on the day following extinction training. Additionally, these results are also consistent with previous studies showing that LTP-like response occurred in the PL after extinction training (Herry et al, 1999; Herry and Garcia, 2002; Farinelli et al, 2006; Hugues et al, 2006, 2007). Moreover, Herry and Garcia (2002) found a correlation between extinction of toneelicited fear and enhanced synaptic plasticity in the mediodorsal thalamus-PL projections. However, future studies should further elucidate the distinction between the PL and the IL and their relevance to fear and extinction.

It is noteworthy that the changes in the EFPs following extinction in both pathways preceded behavioral changes, suggesting that the plastic changes are required to initiate the behavior. This observation is supported by previous findings in the lateral amygdala and the hippocampus showing that changes in neuronal firing and plasticity preceded behavior transition (Berger and Thompson, 1982; Repa et al, 2001). It could be interesting in the future to address the effects of the alteration of early plastic changes on subsequent behavior.

\section{Reinstatement of Fear in the mPFC-BLA Circuit}

To confirm the plastic nature of the circuit, we studied the effect of fear reinstatement following extinction training. In the mPFC-BLA pathway, EFPs amplitude was increased following the return of fear. The positive and strong correlation observed between freezing levels and EFPs re-potentiation following reinstatement, strengthens our idea that indeed fear is mediated by potentiation in the EFPs in the BLA.

In contrast to the conditioning protocol, fear reinstatement was associated with significant depotentiation of the BLA-mPFC pathway. This suggests that previous fear learning might have altered durably the circuit through metaplasticity changes and enabling it to express reduction 
in the EFPs. Moreover, the strong negative correlation between fear freezing recovery and the magnitude of the depotentiation indicates that reinstatement of fear can result in robust fear recovery, impaired extinction, and reduction in the EFPs amplitude.

\section{The Interaction Between the mPFC and the BLA: Relevance to Extinction}

Overall, the results of this study show that the circuit of mPFC-BLA undergoes dynamic and plastic changes following fear and extinction of fear.

Our data pinpoint the differential and opposite synaptic changes in a circuit crucial for fear and extinction learning (Morgan et al, 1993; Milad and Quirk, 2002; Royer and Paré, 2002; Rosenkranz et al, 2003; Likhtik et al, 2005).

It was previously reported that degree of extinction success is correlated with high-frequency bursting in IL neurons immediately after extinction training (BurgosRobles et al, 2009). Bursting in IL could increase local calcium currents as well as depolarization and calcium entry in downstream targets of IL, which would favor the development of extinction-related plasticity. This extinction-related bursting could induce potentiation of synaptic inputs (Herry and Garcia, 2002), and/or increases in intrinsic excitability in the IL (Santini et al, 2008). The generation of LTP-like in the mPFC might constitute a mechanism that enables the consolidation of extinction (Herry and Garcia, 2002) by decreasing the excitatory flow to the BLA. Thus, the decrease in the excitatory flow to the BLA may be reflected in LTD-like processes; this could be achieved via neurons in the BLA that respond to low fear levels. Herry et al (2008) showed in the basal amygdala neurons bidirectional transitions between states of high and low fear, which trigger a rapid switch in the balance of activity between two distinct populations. The investigators report that extinction neurons, in turn, are bi-directionally connected with the mPFC and are switched on during extinction training, indicating that they may be upstream of mPFC neurons thought to mediate consolidation of extinction memory (Milad and Quirk, 2002).

For example, it may be argued that learning-induced alterations in the EFPs (potentiation/depression) observed in our study are mediated by different types of neuronal sub-population in the BLA. However, given the limitations of the EFPs technique and the fact that the BLA and the $\mathrm{mPFC}$ are not laminar structures, we cannot differentiate between different neuronal sub-populations. Furthermore, we cannot exclude that a portion of the stimulated axons will depend on local integration of inputs in the cell bodies, for example, suggesting that the changes that were observed in the BLA could be directly related to the changes in the MPFC and vice versa.

To summarize, in this work we have shown that FC and extinction of fear are regulated via differential and opposite modifications in the EFPs amplitudes in the mPFC-BLA circuit. The reversal from LTP-like to LTD-like, enables a switch and the execution of the appropriate behavior and could constitute a physiological signature for fear and extinction. A deficit in this reversal-switching strategy could be the basis for maladaptive behavior.

\section{ACKNOWLEDGEMENTS}

We thank Professor Rene Garcia for his critical and valuable comments on the MS. This work was supported by the Israel Science Foundation (ISF, grant \# 469/07) to MM.

\section{DISCLOSURE}

The authors declare no conflict of interest.

\section{REFERENCES}

Akirav I, Maroun M (2007). The role of the medial prefrontal cortex-amygdala circuit in stress effects on the extinction of fear. Neural Plast 2007: 30873.

Akirav I, Raizel H, Maroun M (2006). Enhancement of conditioned fear extinction by infusion of the GABA(A) agonist muscimol into the rat prefrontal cortex and amygdala. Eur J Neurosci 23: 758-764.

Akirav I, Segev A, Motanis H, Maroun M (2009). D-cycloserine into the BLA reverses the impairing effects of exposure to stress on the extinction of contextual fear, but not conditioned taste aversion. Learn Mem 16: 682-686.

Berger TW, Thompson RF (1982). Hippocampal cellular plasticity during extinction of classically conditioned nictitating membrane behavior. Behav Brain Res 4: 63-76.

Brambilla R, Gnesutta N, Minichiello L, White G, Roylance AJ, Herron CE et al (1997). Role for the Ras signaling pathway in synaptic transmission and long-term memory. Nature 390: 281-286.

Burgos-Robles A, Vidal-Gonzalez I, Quirk GJ (2009). Sustained conditioned responses in prelimbic prefrontal neurons are correlated with fear expression and extinction failure. $J$ Neurosci 29: 8474-8482.

Fanselow MS, LeDoux JE (1999). Why we think plasticity underlying Pavlovian fear conditioning occurs in the basolateral amygdala. Neuron 23: 229-232.

Farinelli M, Deschaux O, Hugues S, Thevenet A, Garcia R (2006). Hippocampal train stimulation modulates recall of fear extinction independently of prefrontal cortex synaptic plasticity and lesions. Learn Mem 13: 329-334.

Garcia R, Paquereau J, Vouimba RM, Jaffard R (1998). Foot-shock stress but not contextual fear conditioning induces longterm enhancement of auditory-evoked potentials in the basolateral amygdala of the freely behaving rat. Eur J Neurosci 10: 457-463.

Garcia R, Spennato G, Nilsson-Todd L, Moreau JL, Deschaux O (2008). Hippocampal low-frequency stimulation and chronic mild stress similarly disrupt fear extinction memory in rats. Neurobiol Learn Mem 89: 560-566.

Garcia R, Vouimba RM, Baudry M, Thompson RF (1999). The amygdala modulates prefrontal cortex activity relative to conditioned fear. Nature 402: 294-296.

Herry C, Ciocchi S, Senn V, Demmou L, Müller C, Lüthi A (2008). Switching on and off fear by distinct neuronal circuits. Nature 454: 600-606.

Herry C, Garcia R (2002). Prefrontal cortex long-term potentiation, but not long-term depression, is associated with the maintenance of extinction of learned fear in mice. J Neurosci 22: 577-583.

Herry C, Vouimba RM, Garcia R (1999). Plasticity in the mediodorsal thalamo-prefrontal cortical transmission in behaving mice. J Neurophysiol 82: 2827-2832.

Hikind N, Maroun M (2008). Microinfusion of the D1 receptor antagonist, SCH23390 into the IL but not the BLA impairs consolidation of extinction of auditory fear conditioning. Neurobiol Learn Mem 90: 217-222. 
Hugues S, Chessel A, Lena I, Marsault R, Garcia R (2006). Prefrontal infusion of PD098059 immediately after fear extinction training blocks extinction-associated prefrontal synaptic plasticity and decreases prefrontal ERK2 phosphorylation. Synapse 60: 280-287.

Hugues S, Garcia R, Léna I (2007). Time course of extracellular catecholamine and glutamate levels in the rat medial prefrontal cortex during and after extinction of conditioned fear. Synapse 61: 933-937.

Izquierdo A, Wellman CL, Holmes A (2006). Brief uncontrollable stress causes dendritic retraction in infralimbic cortex and resistance to fear extinction in mice. J Neurosci 26: 5733-5738.

Judo C, Matsumoto M, Yamazaki D, Hiraide S, Yanagawa Y, Kimura $S$ et al (2010). Early stress exposure impairs synaptic potentiation in the rat medial prefrontal cortex underlying contextual fear extinction. Neuroscience 169: 1705-1714.

Kavushansky A, Vouimba RM, Cohen H, Richter-Levin G (2006). Activity and plasticity in the CA1, the dentate gyrus, and the amygdala following controllable vs uncontrollable water stress. Hippocampus 16: 35-42.

Kim SC, Jo YS, Kim IH, Kim H, Choi JS (2010). Lack of medial prefrontal cortex activation underlies the immediate extinction deficit. J Neurosci 30: 832-837.

Koseki H, Matsumoto M, Togashi H, Miura Y, Fukushima K, Yoshioka M (2009). Alteration of synaptic transmission in the hippocampal-mPFC pathway during extinction trials of contextdependent fear memory in juvenile rat stress models. Synapse 63: 805-813.

LeDoux JE (2000). Emotion circuits in the brain. Annu Rev Neurosci 23: 155-184.

Likhtik E, Pelletier JG, Paz R, Paré D (2005). Prefrontal control of the amygdala. J Neurosci 25: 7429-7437.

Lin CH, Lee CC, Gean PW (2003). Involvement of a calcineurin cascade in amygdala depotentiation and quenching of fear memory. Mol Pharmacol 63: 44-52.

Maren S (1999). Long-term potentiation in the amygdala: a mechanism for emotional learning and memory. Trends Neurosci 22: $561-567$.

Maroun M (2006). Stress reverses plasticity in the pathway projecting from the ventromedial prefrontal cortex to the basolateral amygdala. Eur J Neurosci 24: 2917-2922.

Maroun M, Richter-Levin G (2003). Exposure to acute stress blocks the induction of long-term potentiation of the amygdalaprefrontal cortex pathway in vivo. J Neurosci 23: 4406-4409.

McKernan MG, Shinnick-Gallagher P (1997). Fear conditioning induces a lasting potentiation of synaptic currents in vitro. Nature 390: 607-611.

Milad MR, Quirk GJ (2002). Neurons in medial prefrontal cortex signal memory for fear extinction. Nature 7: 70-74.

Morgan MA, Romanski LM, LeDoux JE (1993). Extinction of emotional learning: contribution of medial prefrontal cortex. Neurosci Lett 26: 109-113.

Motanis H, Maroun M (2010). Exposure to a novel context following contextual fear conditioning enhances the induction of hippocampal long-term potentiation. Eur J Neurosci 32: 840-846.

Paxinos G, Watson C (1998). The Rat Brain in Stereotaxic Coordinates. Academic Press: San Diego, CA.

Quirk GJ, Likhtik E, Pelletier JG, Pare D (2003). Stimulation of medial prefrontal cortex decreases the responsiveness of central amygdala output neurons. J Neurosci 23: 8800-8807.

Repa JC, Muller J, Apergis J, Desrochers TM, Zhou Y, LeDoux JE (2001). Two different lateral amygdala cell populations contribute to the initiation and storage of memory. Nat Neurosci 4: 724-731.

Richter-Levin G, Maroun M (2010). Stress and amygdala suppression of metaplasticity in the medial prefrontal cortex. Cereb Cortex 20: 2433-2441.

Rocher C, Spedding M, Munoz C, Jay TM (2004). Acute stressinduced changes in hippocampal/prefrontal circuits in rats: effects of antidepressants. Cereb Cortex 14: 224-229.

Rodríguez Manzanares PA, Isoardi NA, Carrer HF, Molina VA (2005). Previous stress facilitates fear memory, attenuates GABAergic inhibition, and increases synaptic plasticity in the rat basolateral amygdala. J Neurosci 25: 8725-8734.

Rogan MT, Leon KS, Perez DL, Kandel ER (2005). Distinct neural signatures for safety and danger in the amygdala and striatum of the mouse. Neuron 46: 309-320.

Rogan MT, Stäubli UV, LeDoux JE (1997). Fear conditioning induces associative long-term potentiation in the amygdala. Nature 390: 604-607.

Royer S, Paré D (2002). Bidirectional synaptic plasticity in intercalated amygdala neurons and the extinction of conditioned fear responses. Neuroscience 115: 455-462.

Rosenkranz JA, Moore H, Grace AA (2003). The prefrontal cortex regulates lateral amygdala neuronal plasticity and responses to previously conditioned stimuli. J Neurosci 23: 11054-11064.

Santini E, Quirk GJ, Porter JT (2008). Fear conditioning and extinction differentially modify the intrinsic excitability of infralimbic neurons. J Neurosci 28: 4028-4036.

Sotres-Bayon F, Quirk GJ (2010). Prefrontal control of fear: more than just extinction. Curr Opin Neurobiol 20: 231-235.

Storsve AB, McNally GP, Richardson R (2010). US habituation, like CS extinction, produces a decrement in conditioned fear responding that is NMDA dependent and subject to renewal and reinstatement. Neurobiol Learn Mem 93: 463-471.

Tang J, Wotjak CT, Wagner S, Williams G, Schachner M, Dityatev A (2001). Potentiated amygdaloid auditory-evoked potentials and freezing behavior after fear conditioning in mice. Brain Res 919: 232-241.

Vidal-Gonzalez I, Vidal-Gonzalez B, Rauch SL, Quirk GJ (2006). Microstimulation reveals opposing influences of prelimbic and infralimbic cortex on the expression of conditioned fear. Learn Mem 13: 728-733.

Vouimba RM, Yaniv D, Diamond D, Richter-Levin G (2004). Effects of inescapable stress on LTP in the amygdala $v s$ the dentate gyrus of freely behaving rats. Eur J Neurosci 19: 1887-1894. 